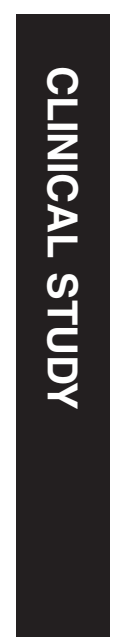

\section{OCT angiography and sequential quantitative analysis of type 2 neovascularization after ranibizumab therapy}

${ }^{1}$ Doheny Eye Institute, Los Angeles, CA, USA

${ }^{2}$ Stein Eye Institute, Los Angeles, CA, USA

${ }^{3}$ Department of Ophthalmology, David Geffen School of Medicine at UCLA, Los Angeles, CA, USA

${ }^{4}$ Greater Los Angeles VA Healthcare Center, Los Angeles, CA, USA

Correspondence:

D Sarraf, Retinal Disorders and Ophthalmic Genetics Division, Stein Eye Institute, David Geffen School of Medicine, University of California, Los Angeles, California, USA

Tel: +1 310794 9921;

Fax: +1 3107947904 .

E-mail: dsarraf@ucla.edu

Received: 12 February 2015 Accepted in revised form: 9 April 2015

Published online:

15 May 2015

The work has not previously been presented at a meeting.

\begin{abstract}
Purpose To study the precise structural aspects of a type 2 neovascular membrane in a patient with age-related macular degeneration (AMD) using optical coherence tomography (OCT) angiography and perform sequential quantitative analysis of the membrane after ranibizumab therapy.

Patients and methods Split-spectrum amplitude-decorrelation (SSADA) OCT angiography macular cubes $(3 \times 3 \mathrm{~mm})$ were acquired with a light source centered at $840 \mathrm{~nm}$, a bandwidth of $45 \mathrm{~nm}$, and an A-scan-rate of 70000 scans per second. Visible pathologic vessels were outlined manually on average intensity projection en face images, and the area of the lesion and the vessel density were measured at baseline and follow-up.

Results At baseline, the neovascular lesion measured $4.12 \mathrm{~mm}^{2}$ and the vessel density was $19.83 \mathrm{~mm}^{-1}$. Four weeks after the first, and 2 and 4 weeks after the second ranibizumab injection, OCT angiography revealed a progressively smaller vascular lesion $\left(2.32,1.77\right.$ and $\left.1.64 \mathrm{~mm}^{2}\right)$, and vessel density $\left(10.24,8.52\right.$ and $\left.7.57 \mathrm{~mm}^{-1}\right)$, although the large central trunks of the lesion were unchanged.

Conclusions In this study, an obvious reduction in size and vessel density of the neovascular lesion was noted after treatment with ranibizumab using SSADA OCT angiography technology. Microvascular components can be delineated with precision, suggesting that this technique may be useful for the management of patients with neovascular AMD in a clinical setting as well as for future clinical trials.
\end{abstract}

L Kuehlewein ${ }^{1,2}$, SR Sadda ${ }^{1,2}$ and D Sarraf ${ }^{2,3,4}$

Eye (2015) 29, 932-935; doi:10.1038/eye.2015.80; published online 15 May 2015

Introduction

Optical coherence tomography (OCT)

angiography is a novel technology that rapidly and conveniently images blood vessels in vivo without the use of exogenous dyes, providing high-resolution and three-dimensional information on the morphology of physiological and pathological blood vessels at different layers of the retina by separating static (tissue) from motion (blood flow) signals. ${ }^{1-3}$ For this purpose, phase- and amplitude-based OCT angiography algorithms have been developed. Algorithms calculating differences in phase are susceptible for noise caused by the OCT system and motion artifacts. Those can be minimized with amplitude-based OCT angiography algorithms, calculating differences in intensity. Splitspectrum amplitude-decorrelation angiography (SSADA) is an improved amplitude-based algorithm that has been introduced to further improve the signal-to-noise ratio by generating multiple repeat OCT frames from two original repeat OCT frames by splitting the spectrum. ${ }^{4}$

OCT angiography has been employed to study the neovascular complex in age-related macular degeneration (AMD). ${ }^{2,3}$ Type 2 neovascularization in AMD originates from the choroid, but is present above the retinal pigment epithelium in the subretinal compartment. ${ }^{5}$ In this study, we employed SSADA technology to study a type 2 neovascular membrane before and after ranibizumab therapy in a patient with AMD. 


\section{Materials and methods}

This case study complied with Institutional Review Board regulations and the requirements of the Declaration of Helsinki.

OCT angiography images were acquired using the RTVue XR Avanti with AngioVue (Optovue Inc., Fremont, CA, USA), with a light source centered at $840 \mathrm{~nm}$, a bandwidth of $45 \mathrm{~nm}$, and an A-scan-rate of 70000 scans per second. Macular cubes $(3 \times 3 \mathrm{~mm})$ were acquired, each cube consisting of 304 clusters of two repeated B-scans containing 304 A-scans each. SSADA technology was employed to improve the signal-to-noise ratio by generating multiple repeat OCT frames from two original repeat OCT frames by splitting the spectrum. ${ }^{4}$ Motion correction was performed using registration of two orthogonally captured imaging volumes. 6,7 To delineate the plane to visualize the neovascular membrane, the automated segmentation lines were adjusted to the inner and outer margin of the lesion. En face images of the vasculature were generated by average intensity projection for the identified layer. Quantitative analyses of the neovascular membrane were performed using the publically available GNU Image Manipulation Program GIMP 2.8.14 (http:/ / gimp.org) by manually outlining visible vessels with a 1 pixel wide line. For each visit, the area of the membrane was assessed in pixels and converted to $\mathrm{mm}^{2}$ using the following equation: lesion area $\left(\mathrm{mm}^{2}\right)=$ lesion area $(\mathrm{px}) \times(3 \mathrm{~mm} / 304 \mathrm{px})^{2}$. The vessel density was assessed as the total vessel length per baseline lesion area in $\mathrm{mm}^{-1}$.

\section{Results}

An 89-year-old African-American male with a history of neovascular AMD presented with decreased vision in the right eye for 2 weeks. Both eyes had been treated with intravitreal injections of ranibizumab over 1 year before presentation. The left eye had evolved to an end-stage disciform scar despite multiple ranibizumab injections.
At examination, the patient's visual acuity was 20/300 in the right eye, and count fingers in the left eye. Dilated fundus examination showed macular hemorrhage in the right eye and an end-stage disciform scar in the left eye (Figure 1). Spectral domain OCT imaging of the right eye revealed a shallow fibrovascular pigment epithelial detachment with overlying type 2 neovascularization and associated subretinal fluid. Fluorescein angiography showed a classic neovascular membrane with leakage that corresponded to the type 2 membrane on spectral domain OCT imaging (Figure 1). OCT angiography showed a large type 2 neovascular lesion with two large caliber central trunks or feeder vessels, one superior and one inferior, and a branching network of dense smaller caliber vessels radiating in all directions from the main trunk. Quantitative OCT angiography analysis of the area of the microvascular lesion measured $4.12 \mathrm{~mm}^{2}$, and the vessel density was $19.83 \mathrm{~mm}^{-1}$ at baseline (Figure 2, Table 1).

A series of ranibizumab injections was initiated the following day, and 4 and 8 weeks later. Four weeks after the first injection of the series, the patient's visual acuity had improved to $20 / 80$ in the right eye, and OCT angiography revealed a smaller vascular lesion area $\left(2.32 \mathrm{~mm}^{2}\right)$ with a decreased vessel density of $10.24 \mathrm{~mm}^{-1}$, but the large central trunks were unchanged. Two and four weeks after the second injection, OCT angiography revealed a progressively smaller vascular lesion area and reduced vessel density (Figure 2, Table 1).

\section{Discussion}

Herein, we present the case of a patient with type 2 neovascularization and AMD in the right eye in whom sequential OCT angiography was performed.

Quantitative analysis of the neovascular membrane was done at baseline and at designated intervals after ranibizumab therapy.

Four weeks after the first ranibizumab injection, the area of the neovascular membrane was significantly reduced, by $\sim 40 \%$, whereas the associated dense vascular
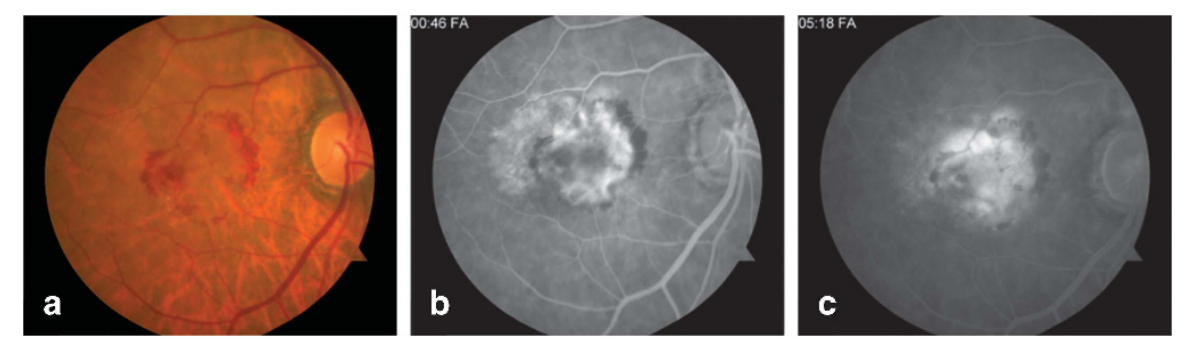

Figure 1 (a) Color fundus photograph of the right eye showing macular hemorrhage. (b and c) Early and late frames of a fluorescein angiography showing classic type 2 neovascularization with central leakage and an adjacent pigment epithelium detachment at the temporal margin. 

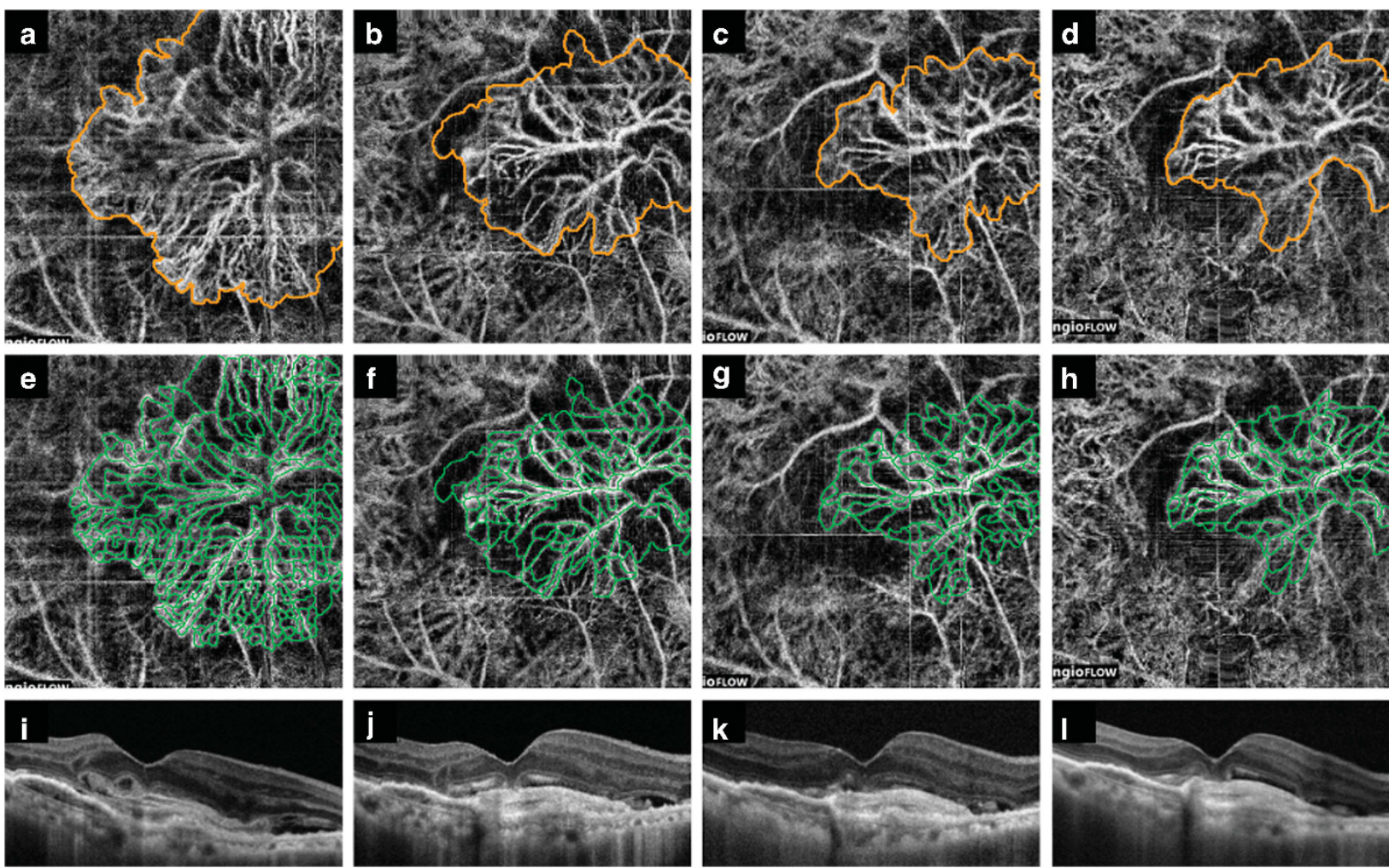

Figure $2(\mathrm{a}-\mathrm{h})$ OCT angiography of type 2 neovascularization of the right eye at baseline and at intervals after ranibizumab therapy. (a-d) OCT angiography of type 2 neovascularization with margins outlined at baseline and at 4-week and then 2-week intervals. Note the progressive reduction in the size of the membrane. (e-h) OCT angiography of type 2 neovascularization with color-coded capillary density analysis at baseline and at aforementioned follow-up intervals. Note the progressive reduction in capillary density of the membrane. (i-1) Corresponding spectral domain OCT scans at baseline and follow-up intervals after ranibizumab therapy.

Table 1 Area of type 2 neovascularization and associated vessel density at baseline and at designated follow-up intervals after ranibizumab injection

\begin{tabular}{lrrrc}
\hline & Baseline & Week 4 & Week 6 & Week 8 \\
\hline Lesion area $\left(\mathrm{mm}^{2}\right)$ & 4.12 & 2.32 & 1.77 & 1.64 \\
Vessel density $\left(\mathrm{mm}^{-1}\right)$ & 19.83 & 10.24 & 8.52 & 7.57 \\
\hline
\end{tabular}

plexus was also remarkably attenuated by $\sim 50 \%$. Four weeks after the second injection, the lesion size had further decreased, by $\sim 30 \%$, whereas the vascular plexus was attenuated by $\sim 25 \%$. This progressive reduction in the size (ie, area) and density of the neovascular complex is well illustrated in Figure 2.

Although the type 2 neovascular membrane was progressively reduced in size and density, the main central trunk of feeder vessels remained unchanged. Feeder vessels have been a notable constituent of neovascular membranes. ${ }^{8}$ It has been suggested that the main feeder vessel or central trunk may be more resistant to anti-VEGF therapy as the endothelial cells are protected by overlying pericytes whereas the branching vascular network consists of unprotected endothelial cells that are more responsive to anti-VEGF therapy. ${ }^{9,10}$

OCT angiography is still fraught with important limitations including motion and projection artifact and insufficient temporal resolution and sensitivity to detect low flow or small caliber lesions. In our study, the type 2 neovascular complex was significantly attenuated after anti-VEGF therapy, which may be the result of undetectable lower flow signals or frankly regressed vessels.

In summary, OCT angiography provides for the first time detailed imaging of the vascular architecture of neovascular membranes in AMD. We studied the precise structural aspects of a type 2 neovascular complex using OCT angiography and were able to perform sequential quantitative analysis of the membrane after ranibizumab therapy. Obvious reduction in the size and density of the neovascular lesion was noted. Vascular components can be delineated with precision using SSADA OCT angiography technology, suggesting that this technique may be useful for managing patients with neovascular AMD and assessing potential treatments for this disease in future clinical trials. 


\section{Summary}

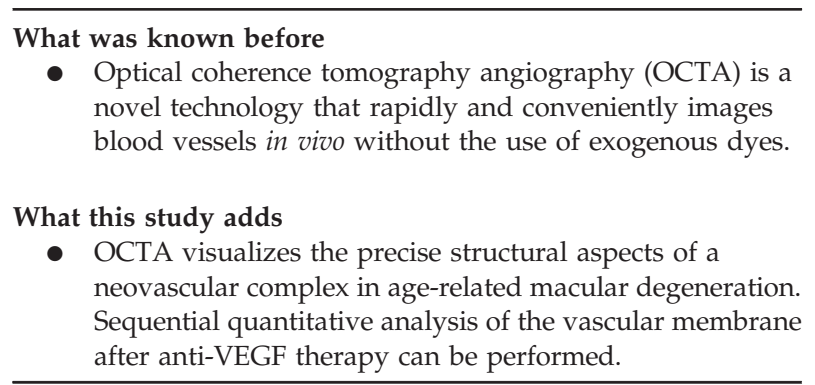

\section{Conflict of interest}

Dr Kuehlewein declares no potential conflict of interest. Dr Sadda receives research support from and serves as a consultant for Genentech. The Optovue OCT machine was loaned to Dr Sarraf for research purposes. Dr Sarraf has research grants from Regeneron and Genentech.

\section{Acknowledgements}

Dr Sadda is a co-inventor of Doheny intellectual property related to optical coherence tomography that has been licensed by Topcon Medical Systems and is a member of the scientific advisory board for Heidelberg Engineering. Dr Sadda receives research support from and serves as a consultant for Carl Zeiss Meditec, Optos, and Allergan. He also serves as a consultant for Alcon, Novartis, and Iconic.

\section{References}

1 Spaide RF, Klancnik Jr JM, Cooney MJ. Retinal vascular layers imaged by fluorescein angiography and optical coherence tomography angiography. JAMA Ophthalmol 2015; 133: 45-50.

2 Jia Y, Bailey ST, Wilson DJ, Tan O, Klein ML, Flaxel CJ et al. Quantitative optical coherence tomography angiography of choroidal neovascularization in age-related macular degeneration. Ophthalmology 2014; 121: 1435-1444.

3 Moult E, Choi W, Waheed NK, Adhi M, Lee B, Lu CD et al. Ultrahigh-speed swept-source OCT angiography in exudative AMD. Ophthalmic Surg Lasers Imaging Retina 2014; 45: 496-505.

4 Jia Y, Tan O, Tokayer J, Potsaid B, Wang Y, Liu JJ et al. Split-spectrum amplitude-decorrelation angiography with optical coherence tomography. Opt Express 2012; 20: $4710-4725$.

5 Freund KB, Zweifel SA, Engelbert M. Do we need a new classification for choroidal neovascularization in age-related macular degeneration? Retina 2010; 30: 1333-1349.

6 Kraus MF, Potsaid B, Mayer MA, Bock R, Baumann B, Liu JJ et al. Motion correction in optical coherence tomography volumes on a per A-scan basis using orthogonal scan patterns. Biomed Opt Express 2012; 3: 1182-1199.

7 Kraus MF, Liu JJ, Schottenhamml J, Chen CL, Budai A, Branchini L et al. Quantitative 3D-OCT motion correction with tilt and illumination correction, robust similarity measure and regularization. Biomed Opt Express 2014; 5: 2591-2613.

8 Kozak I, Cheng L, Cochran DE, Freeman WR. Phase I clinical trial results of verteporfin enhanced feeder vessel therapy in subfoveal choroidal neovascularisation in age related macular degeneration. Br J Ophthalmol 2006; 90: 1152-1156.

9 Bellou S, Pentheroudakis G, Murphy C, Fotsis T. Anti-angiogenesis in cancer therapy: hercules and hydra. Cancer Lett 2013; 338: 219-228.

10 Benjamin LE, Hemo I, Keshet E. A plasticity window for blood vessel remodelling is defined by pericyte coverage of the preformed endothelial network and is regulated by PDGF-B and VEGF. Development 1998; 125: 1591-1598. 